Fabienne D. Schwab*, Eva K. Zettler, Andala Moh, Andreas Schötzau, Uwe Gross

and Andreas R. Günthert

\title{
Predictive factors for preterm delivery under rural conditions in post-tsunami Banda Aceh
}

\begin{abstract}
Objective: To assess the risk for preterm deliveries $<37$ week of gestation and associated prevalence of vaginal infection in a rural setting after the tsunami in Banda Aceh, Indonesia.

Methods: Wet mount microscopy, vaginal $\mathrm{pH}$ and vaginal swabs for microbiological culture were collected in pregnant women during the $2^{\text {nd }}$ trimester from February to June of 2005 in four temporary outpatient clinics and the patients were followed up until delivery.

Results: One hundred and fifty-nine pregnant patients were screened. Sixty-two could be followed up until delivery. Thirty-nine (62.9\%) delivered at term and $23(37.1 \%)$ delivered prematurely. Significant risk factors for preterm delivery were a history of preterm delivery and group B streptococcus infection. Increased vaginal $\mathrm{pH}$ alone had no significant influence on preterm delivery, although there was a trend.

Conclusion: The rate of preterm delivery was high in this cohort. We suggest risk stratification for preterm delivery in rural conditions by performing a vaginal $\mathrm{pH}$ and wet mount microscopy. If either is suspect we suggest collecting a vaginal swab for microbiological culture for targeted treatment. Patients with a history of preterm delivery are at increased risk and should be monitored closely.
\end{abstract}

Keywords: Natural disaster; preterm delivery; rural setting; vaginal infection; wet mount microscopy.

\footnotetext{
*Corresponding author: Fabienne D. Schwab, Department of Obstetrics and Gynaecology, University Hospital of Basel, Basel, Switzerland, E-mail: fabienne.schwab@usb.ch

Andreas Schötzau: Department of Obstetrics and Gynaecology, University Hospital of Basel, Basel, Switzerland

Eva K. Zettler: Department of Surgery, Klinikum am Urban, Berlin, Germany

Andala Moh: Department of Obstetrics and Gynaecology, Syiah Kuala University, Banda Aceh, Indonesia

Uwe Gross: Institute of Medical Microbiology, Göttingen University Medical Centre, Göttingen, Germany

Andreas R. Günthert: Department of Obstetrics and Gynaecology, Cantonal Hospital of Lucerne, Lucerne, Switzerland
}

DOI 10.1515/jpm-2015-0004

Received January 3, 2015. Accepted April 17, 2015. Previously published online May 14, 2015.

\section{Introduction}

Preterm delivery is a leading cause of neonatal morbidity and mortality [1]. Preterm birth contributes significantly to newborn morbidity, including neonatal complications, cerebral palsy, cognitive impairment, blindness, deafness and respiratory illness [2]. There are several risk factors of preterm labour and preterm delivery. Major risk factors for preterm delivery include previous pregnancy with a preterm delivery, Black maternal race, genitourinary infections, smoking, extremes of body weight, social disadvantage, maternal depression, assisted fertility, stress in pregnancy, poor diet and periodontal disease [3]. Bacterial vaginosis is also a well-known risk factor for preterm delivery [3-5]. An elevated vaginal $\mathrm{pH}$ is also a risk factor for adverse pregnancy outcome [6].

There is little data about the prevalence of preterm delivery and the presence of vaginal infection in an isolated population that has just experienced a natural disaster, which has low income and little medical support.

This study was conducted to determine the prevalence and incidence of bacterial infection shortly after the tsunami in an isolated population north of Sumatra in Banda Aceh, Indonesia. Banda Aceh was isolated for more than 10 years due to civil war before the tsunami. As hundreds of thousands of Acehnese recovered from the devastating Indian Ocean tsunami of 26 December 2004, the deadly separatist conflict in Indonesia's northwest came to a long-awaited end. Although unofficial talks were set in motion during 2004, the tsunami was a catalyst, urging a peaceful resolution of the conflict [7, 8].

The inhabitants of Banda Aceh are strict Muslims and sexual intercourse before marriage is prohibited. After the tsunami, the living situation was disastrous for the people. Most of them were homeless, lived in tents or in self-constructed houses. There was no proper sanitation. Most of the patients lost family members, friends or neighbours. The conditions were very rural and while medical 
care existed, it was very limited, as all the hospitals and outpatient clinics were at least partly destroyed. Antenatal care was predominantly done by midwifes or nurses.

The aim of this study was to determine if patient history, wet mount microscopy and vaginal $\mathrm{pH}$ are of predictive value in this cohort for preterm delivery. Furthermore, the purpose of this study was to determine if microbiologic testing is of value for predicting preterm delivery and if the $\mathrm{pH}$ and/or wet mount microscopy correlated with vaginal infection. This study represents a population in a very rural setting with very limited medical support comparable to first aid refugee camps in a tropical area. This study should enable an algorithm for risk stratification for preterm delivery in rural conditions, such as in disasters, for people on migration and people in developed countries.

To our knowledge, our study is the first comprehensive analysis that examined the preterm risk and prevalence of vaginal infection in a very rural, isolated setting after a natural disaster.

\section{Material and methods}

After the tsunami, wet mount microscopy, vaginal $\mathrm{pH}$ and vaginal swabs for microbiological culture were collected from pregnant women during the $2^{\text {nd }}$ trimester of pregnancy in four outpatient clinics in Banda Aceh by a trained medical student from February 2005 until June 2005. The wet mount microscopy and $\mathrm{pH}$ were analysed immediately. Cultures for Candida albicans and group B Streptococcus were also done on site. Group B Streptococcus cultures were tested in every pregnant woman that entered the outpatient clinic by a vaginal swap. We did not perform a risk-based approach and screened every woman, not just those between 35 and 37 weeks of gestation. Vaginal swabs for Ureaplasma urealyticum, Mycoplasma hominis, Chlamydia trachomatis and Neisseria gonorrhoea were collected, stored and sent to the microbiology laboratory at the University of Göttingen, Göttingen, Germany, for analysis with the LightCycler 1.5 Real-Time PCR system (Roche Diagnostics Deutschland GmbH, Mannheim, Germany). Results of the microbiological analysis were received after a time delay and had no impact on any specific treatment decisions. Vaginal $\mathrm{pH}>4.5$ was defined as pathological. Wet mount microscopy was defined as abnormal or positive for infection when there was the presence of clue cells, leucocytes or yeast (pseudohyphal or true hyphae forms). Bacterial vaginosis was defined as clue cells, leucocytes on microscopy and vaginal $\mathrm{pH}>4.5$. The students' immediate results of wet mount and $\mathrm{pH}$ in asymptomatic women had no impact on decision making of midwifes or physicians, as these methods were not part of standard care and not accepted as a specific screening method. There was a loss of follow up with the majority of patients as many of them moved away from the place of disaster to other cities or islands; in addition, some delivered at home. In some cases, delivery data could be collected retrospectively by a local gynaecologist who called up patients during the years of reconstruction after the tsunami. He was also able to collect data during postpartum follow up visits. The data collection methods and study design were approved by the Ethics Committee of the University of Göttingen. Oral informed consent was obtained from all women.

\section{Statistical methods}

Descriptive statistic is presented as means (standard deviation) or counts and percentages as appropriate. In order to compare metric variables, $t$-tests were calculated. Comparisons between categorical variables were made using Fishers exact-tests. In order to predict "preterm delivery < 37 weeks of gestation" or "infection" univariable logistic regression was performed. Results are presented as odds ratios (OR) with corresponding 95\% confidence intervals (CI) and $\mathrm{P}$-values. In the case of metric variables, results are presented as the ratio of the odds increasing the predictor one unit. OR could not be estimated for all predictors, therefore, only P-values of the likelihood ratio test was presented. A P-value $<0.05$ was considered as significant. We did not adjust for confounders because the sample size was too small. All evaluations were performed with R Development Core Team software, version 3.0.1 [R Foundation for Statistical Computing, Vienna, Austria. http://R-project.org/].

\section{Results}

One hundred and fifty-nine pregnant patients participated in the study. Sixty-two could be followed up until delivery. Gestational age was based on self-reported last menstruation. Thirty-nine patients $(62.9 \%)$ delivered at term, 19 (30.6\%) delivered between 34 and 37 weeks, and four (6.67\%) patients delivered before 34 weeks. The mean age was 25.6 (range 17-42) years for all patients and 26.7 (range 17-41) years for patients with premature delivery. Age was not associated with preterm delivery. Descriptive statistics for all deliveries are presented in Table 1. In our collective, $21.4 \%$ (34/159) were positive on wet mount microscopy and had bacterial vaginosis. Of the 39 patients who delivered at term, five were positive in the wet mount microscopy for infection, four of 19 were positive who delivered between 34 and 37 weeks, and only one of four was positive on wet mount microscopy who had a preterm delivery before 34 weeks.

Nine patients had a history of premature delivery and 12 patients had a history of spontaneous abortion. Twenty-six (16.4\%) were positive for group B Streptococcus in microbiological culture, 81 (50.9\%) were positive for Candida, 32 (20.1\%) were positive for M. hominis and 50 (31.4\%) patients were positive for $U$. urealyticum. Only one $(0.63 \%)$ was positive for $C$. trachomatis and another one was positive for $N$. gonorrhoea.

We tried to determine which risk factors in our patient collective were predictive for preterm delivery (Table 2). If a woman had a history of premature delivery, the risk 
Table 1: Descriptive statistics for patients who delivered based on gestational age at delivery, counts (percentage) and mean (standard deviation).

\begin{tabular}{lrrr}
\hline & $\begin{array}{r}\text { All deliveries } \\
\mathbf{n}=62\end{array}$ & $\begin{array}{r}\text { Term deliveries } \\
\mathbf{n = 3 9}\end{array}$ & $\begin{array}{r}\text { Preterm deliveries <37 } \\
\mathbf{n}=\mathbf{2 3}\end{array}$ \\
\hline Age & 26.6 years & 26.5 years & 26.7 years \\
Infection on wet mount & $10 / 62(16 \%)$ & $5 / 39(13 \%)$ & $5 / 23(22 \%)$ \\
microscopy & & & \\
History of premature delivery & $9 / 62(15 \%)$ & $1 / 39(3 \%)$ & $8 / 23(35 \%)$ \\
History of abortion spontaneous & $8 / 62(13 \%)$ & $5 / 39(13 \%)$ & $3 / 23(13 \%)$ \\
Birth weight & $2860 \mathrm{~g}(518)$ & $3167 \mathrm{~g}(305)$ & $2339 \mathrm{~g}(363)$ \\
Vaginal pH & $4.23(0.64)$ & $4.10(0.67)$ & $4.43(0.57)$ \\
Group B streptococcus infection & $12 / 62(19 \%)$ & $1 / 39(3 \%)$ & $8 / 23(35 \%)$ \\
Candida infection & $33 / 62(53 \%)$ & $21 / 39(57 \%)$ & $12 / 23(52 \%)$ \\
Mycoplasma hominis infection & $13 / 62(21 \%)$ & $11 / 39(28 \%)$ & $2 / 23(8 \%)$ \\
Ureaplsama urealyticum infection & $22 / 62(35 \%)$ & $16 / 39(41 \%)$ & $2 / 23(9 \%)$ \\
Neisseria gonorrhoea infection & $1 / 62(2 \%)$ & $1 / 39(3 \%)$ & - \\
Chlamydia trachomatis infection & $1 / 62(2 \%)$ & - & $1 / 23(4 \%)$ \\
\hline
\end{tabular}

Table 2: Prediction of premature delivery from different parameters.

\begin{tabular}{lrrrrr}
\hline & $\begin{array}{r}\text { Total patients } \\
\mathbf{n}=62\end{array}$ & $\begin{array}{r}\text { Term delivery after 37 } \\
\text { weeks of gestation } \\
\mathbf{n = 3 9}\end{array}$ & $\begin{array}{r}\text { Preterm delivery }<37 \\
\text { weeks of gestation } \\
\mathbf{n}=\mathbf{2 3}\end{array}$ & OR and 95\% Cl & P-ratio \\
\hline Group B streptococcus infection & $12 / 62$ & $4 / 39$ & $8 / 23$ & $4.47(1.19 ; 19.7)$ & 0.026 \\
History of premature delivery & $9 / 62$ & $1 / 39$ & $8 / 23$ & $16.9(2.69 ; 451)$ & 0.001 \\
Vaginal pH & 4.23 & 4.10 & 4.43 & $2.35(0.98 ; 5.66)$ & 0.056 \\
Candida infection & $33 / 62$ & $21 / 39$ & $12 / 23$ & $0.94(0.33 ; 2.68)$ & 0.901 \\
Mycoplasma hominis infection & $13 / 62$ & $11 / 39$ & $2 / 23$ & $0.26(0.03 ; 1.13)$ & 0.075 \\
Ureaplasma urealyticum infection & $22 / 62$ & $16 / 39$ & $6 / 23$ & $0.52(0.15 ; 1.57)$ & 0.251 \\
\hline
\end{tabular}

$\mathrm{OR}=$ odds ratio, $\mathrm{Cl}=$ confidence interval.

for a subsequent preterm delivery was increased [OR 16.9 (2.69-451), $\mathrm{P}=0.001]$. In addition, women with group B Streptococcus infection had an increased risk of premature delivery [OR 4.47 (1.19-19.7), $\mathrm{P}=0.026]$. Sensitivity for preterm delivery by testing for group B Streptococcus was $35 \%$ with a specificity of $90 \%$.

The greater the vaginal $\mathrm{pH}$ was, the higher the risk for preterm delivery. However, the $\mathrm{pH}$ test was not significant, although there was a trend [OR 1.53 (0.99-2.38), $\mathrm{P}=0.056$ ]. All specific infections found in microbiological/PCR tests including Candida, M. hominis and $U$. urealyticum were not significant predictors for preterm delivery.

We examined several parameters to find out whether a specific parameter was predictive for infection on wet mount microscopy. As shown in Table 3, only Candida infection, with an OR of $3.36(1.49 ; 8.24), \mathrm{P}=0.003$, and

Table 3: Prediction of infection from different parameters for all examined pregnant women.

\begin{tabular}{|c|c|c|c|c|c|}
\hline & $\begin{array}{r}\text { Number } \\
\text { total } \\
n=159\end{array}$ & $\begin{array}{r}\text { No infection on wet } \\
\text { mount microscopy } \\
n=125\end{array}$ & $\begin{array}{r}\text { Infection on wet } \\
\text { mount microscopy } \\
n=34\end{array}$ & $\mathrm{OR}$ and $95 \% \mathrm{Cl}$ & P-ratio \\
\hline Group B streptococcus infection & $26 / 159$ & $13 / 125$ & $13 / 34$ & $5.25(2.12 ; 13.2)$ & $<0.001$ \\
\hline Candida infection & $81 / 159$ & $56 / 125$ & $25 / 34$ & $3.36(1.49 ; 8.24)$ & 0.003 \\
\hline Mycoplasma hominis infection & $32 / 159$ & $29 / 125$ & $3 / 34$ & $0.34(0.07 ; 1.04)$ & 0.059 \\
\hline Ureaplasma urealtyticum infection & $50 / 159$ & $38 / 125$ & $12 / 34$ & $1.25(0.55 ; 2.77)$ & 0.587 \\
\hline Neisseria gonorrhoea infection & $1 / 159$ & $1 / 125$ & 0 & n.e & n.e \\
\hline Chlamydia trachomatis infection & $1 / 159$ & $1 / 125$ & 0 & n.e & n.e \\
\hline
\end{tabular}

$\mathrm{OR}=$ odds ratio, $\mathrm{Cl}=$ confidence interval, $\mathrm{n} . \mathrm{e}=$ not estimable. 
group B Streptococcus infection, with an OR of 5.25 (2.12;13.2), $\mathrm{P}<0.001$, were significantly associated with positive wet mount microscopy. All other parameters, such as $U$. urealyticum, $M$. hominis, $C$. trachomatis and $N$. gonorrhoea infection, were not significantly associated with positive wet mount microscopy. The sensitivity of wet mount microscopy for infection with group B Streptococcus was $50 \%$ and the specificity was $84 \%$. The positive predictive value of pathologic wet mount microscopy for group B Streptococcus infections was 0.38 and the negative predictive value was 0.9 .

\section{Discussion}

Our post-tsunami collective had no increased rate of bacterial vaginosis $(21.4 \%)$ compared to pregnant women in other studies [3,9]. As expected in this cohort of patients who were of strict Muslim faith, only one had a C. trachomatis infection and another was positive for $N$. gonorrhoea. This low rate of sexually transmitted infections reflects the sociocultural behaviour of an Islamic population, who lived almost completely isolated due to civil war until the tsunami occurred.

The percentage of preterm delivery was extremely high in our cohort, approximately one-third. In comparison, the WHO lists Indonesia into the top 10 countries of preterm birth. It is estimated that about 15.5 per 100 deliveries are preterm deliveries. Although our follow up data of patients are very limited, the high percentage of preterm delivery reflects the increased risk under such disaster conditions with decreased medical supply and associated stress factors. This increased rate might be partly due to women with preterm delivery being referred to the hospital, rather than delivering at home, and they also had complete follow up. It is possible that many women who delivered at term might have done so at home and they were thus lost to follow up. Therefore, we cannot exclude this potential bias.

As shown in several other studies, high levels of stress have a negative effect on pregnancy, and it is a significant risk factor for preterm birth [2, 10, 11]. Early vulnerability combined with anxiety symptoms in pregnancy confer to increased risk for preterm birth [2]. The high percentage of preterm birth in our collective could be partly explained by the life threatening stress caused by the tsunami. The whole infrastructure was almost completely destroyed. Most patients lost their belongings, family members, friends and colleagues. Some were caught in the current and had to fear for their life. Some saw their family members drowning in the pulling stream of water and the future for many after the tsunami was unclear. All these patients suffered a high level of stress after the Indian Ocean tsunami devastated Aceh's coastal communities and this could be a reason for this high rate of preterm delivery. We collected samples from 159 pregnant patients. Unfortunately, only 62 patients could be followed up until delivery. We lost $61 \%$ of the patient to follow up, as many moved away or delivered at home. The whole infrastructure, including the medical supplies and documentation, was scarce and very limited. Another problem contributing to incomplete data was that most hospitals and outpatient clinics were at least partly destroyed and medical examinations and antenatal care was performed in temporary accommodation. In many cases, documentation of patients' history was lacking or it was not possible to perform properly.

A higher vaginal $\mathrm{pH}$ showed a tendency towards an increased risk for preterm delivery. Significant predictive factors for preterm delivery were the history of premature delivery and group B Streptococcus infection. As we screened every pregnant woman for group B Streptococcus infection, our data confirmed the result of Rausch et al. that a screening-based strategy of group B Streptococcus should be used to prevent neonatal sepsis [12]. Pathologic wet mount microscopy was predictive for infections with group B Streptococcus and/or Candida infection. Until now, there has been no evidence that Candida infection is a risk factor for preterm delivery, which was confirmed in our cohort. In contrast, we found that group B Streptococcus colonization was associated with preterm delivery and might cause preterm labour and/or premature rupture of membranes. There are diverse results in studies that assessed the role of group B Streptococcus colonization in prematurity. Seyyed et al. could show that group B Streptococcus colonization was statistically higher in women with pre-term labour compared with term deliveries [13]. Several other studies suggesting group B Streptococcus as an important contributor to preterm low birth weight, but until now the real impact of group B Streptococcus colonization and preterm delivery remains unclear [14, 15]. Additional large observational studies are required to understand the mechanism that effect group B Streptococcus on pregnancy.

One of the strongest clinical risk factors for preterm birth is a prior preterm birth. Maternal history of preterm birth is commonly reported to confer to a 1.5 -fold-2-fold increased risk in a subsequent pregnancy [3, 16, 17]. As vaginal ultrasound screening in very rural conditions is not available due to lack of knowledge or lack of equipment, follow up for women with a history of preterm birth cannot be the same as in high-income countries with its higher medical standards [3, 17]. 
In conclusion from our data, we suggest an algorithm for risk stratification for preterm delivery in very rural conditions or after natural disasters where medical supply is limited and the usual medical technologies, such as vaginal ultrasound, are not available. We recommend performing a test of vaginal $\mathrm{pH}$ and wet mount microscopy during an antenatal care examination during the second trimester of pregnancy. Wet mount microscopy is not costly to perform and easy to learn, especially since the introduction of the foldscope [18]. The foldscope is an origami-based print-and-fold optical microscope that can be assembled from a flat sheet of paper. If wet mount microscopy shows infection and/or vaginal $\mathrm{pH}$ is higher than 4.5, we suggest taking a vaginal swab for microbiological cultures. If neither vaginal $\mathrm{pH}$ nor wet mount microscopy is pathological, additional microbiological testing is not required. Patients with vaginal infection should be treated adequately and followed up closely as the correlation between bacterial vaginosis and preterm delivery is well known [19]. All patients with a history of preterm delivery should be closely monitored, as a history of preterm delivery is a well-established risk of preterm delivery. If transvaginal ultrasound is available and cervical length is shorter than $20 \mathrm{~mm}$ at 24 weeks of gestation, prophylactic administration with progesterone should be considered in these patients if available $[3,16,17]$. Finally, not only treatment of vaginal infections is important but also psychosocial support of pregnant woman exposed to extraordinary stress circumstances may be helpful for the prevention of preterm delivery $[2,10,11]$. Limitations of our study are the low rate of follow up and the relatively small number of pregnant women. However, the results are conclusive and using wet mount and vaginal $\mathrm{pH}$ in addition to adequate medical history to predict preterm delivery under rural circumstances is feasible.

Acknowledgments: The German Academic Exchange Services in part supported this study. The authors thank Prof. I. Hoesli and Dr. Dorothy Huang for their critical review in the preparation of the original manuscript.

\section{References}

[1] Mendz GL, Kaakoush NO, Quinlivan JA. Bacterial aetiological agents of intra-amniotic infections and preterm birth in pregnant woman. Frontiers Cell Infect Microbiol. 2013;3:58.

[2] McDonald SW, Kingston D, Bayrampour H, Dolan SM, Tough SC. Cumulative psychosocial stress, coping resources, and preterm birth. Arch Womens Ment Health. 2014;17:559-68.
[3] lams JD. Clinical practice. Prevention of preterm parturition. N Engl J Med. 2014;370:254-61.

[4] Brocklehurst P, Gordon A, Heatley E, Milan SJ. Antibiotics for treating bacterial vaginosis in pregnancy. Cochrane Database Syst Rev. 2013;1:CD000262.

[5] Hay PE, Lamont RF, Taylor-Robinson D, Morgan DJ, Ison C, Pearson J. Abnormal bacterial colonisation of the genital tract and subsequent preterm delivery and late miscarriage. $\mathrm{Br}$ Med J. 1994;308:295-8.

[6] Hantoushzadeh S, Sheikh M, Javadian P, Shariat M, Amini E, Abdollahi A, et al. Elevated vaginal $\mathrm{pH}$ in the absence of current vaginal infection, still a challenging obstetrical problem. J Matern Fetal Neonatal Med. 2014;27:582-7.

[7] Zeccola P. Dividing disasters in Aceh, Indonesia: separatist conflict and tsunami, human rights and humanitarianism. Disasters. 2011;35:308-28.

[8] Waizenegger A, Hyndman J. Two solitudes: post-tsunami and post-conflict Aceh. Disasters. 2010;34:787-808.

[9] Islam A, Safdar A, Malik A. Bacterial vaginosis. J Pak Med Assoc. 2009;59:601-4.

[10] Dunkel Schetter C. Psychological science on pregnancy: stress processes, biopsychosocial models, and emerging research issues. Annu Rev Psychol. 2011;62:531-58.

[11] Littleton HL, Bye K, Buck K, Amacker A. Psychosocial stress during pregnancy and perinatal outcomes: a meta-analytic review. J Psychosom Obstet Gynaecol. 2010;31:219-28.

[12] Rausch A, Gross A, Droz S, Bodmer T, Surbek D. Group B Streptococcus colonization in pregnancy: prevalence and prevention strategies of neonatal sepsis. J Perinat Med. 2009;37:124-9.

[13] Seyyed EZ, Toossi, Jalalvand A, Sajadi M. Group B Streptococci investigation in pre-term labors. Med Arch. 2013;67:124-5.

[14] Schuchat A. Epidemiology of group B streptococcal disease in the United States: shifting paradigms. Clin Microbiol Rev. 1998;11:497-513.

[15] Klebanoff MA, Regan JA, Vijaya Rao A, Nugent RP, Blackwelder DA, Eschenbach JG, et al. Outcome of vaginal Infections and Prematurity Study: results of clinical trial of erythryomycin among pregnant women colonized with group B streptococci. Am J Obstet Gynecol. 1995;172: 1540-5.

[16] Slager J, Lynne S. Treatment options and recommendations to reduce preterm birth in woman with short cervix. J Midwifery Womens Health. 2012;57:12-8.

[17] Committee on Practice Bulletins - Obstetrics, The American College of Obstetricians and Gynecologists. Practice bulletin no. 130: prediction and prevention of preterm birth. Obstet Gynecol. 2012;120:964-73.

[18] Cybulski JS, Clements J, Prakash M. Foldscope: origami-based paper microscope. PLoS One. 2014;9:e98781.

[19] Surbek D, Hoesli I, Holzgreve W. Morphology assessed by transvaginal ultrasonography differs in patients in preterm labor with vs. without bacterial vaginosis. Ultrasound Obstet Gynecol. 2000;15:242-5.

The authors stated that there are no conflicts of interest regarding the publication of this article. 